\title{
DECISION SUPPORT APPROACH FOR INTEGRATED MAINTENANCE PROGRAM OF URBAN RAIL TRANSIT
}

\author{
Ming Zhang \\ Institute of Computing Technology, China Academy of Railway Sciences \\ No.2 Daliushu Road, Haidian District, Beijing 100081, China \\ zm_zhangming@hotmail.com
}

\begin{abstract}
Based on the analysis of the complexity of the equipment maintenance business and the correlation characteristics of the monitoring fault of urban rail transit, this paper puts forward the combination of the integrated maintenance process and intelligent maintenance decision method. The selected data is obtained from the relevant system as a basis for preventive maintenance support by introducing the data mining method of the high frequency fault clustering model of electromechanical equipment. Then the decision tree induction strategy is proposed to identify the equipment object-class and rules led to similar fault for different equipment systems. This class serves as a high priority for prevention repair, predict repair, and fault repair, in order to establish the maintenance decision-making program. Then the related method of evaluation and verification are employed. This method is designed and converted as data flow to develop the maintenance management system. The application results show positive effect on reasonable maintenance management. Copyright $₫$ Research Institute for Intelligent Computer Systems, 2017. All rights reserved.
\end{abstract}

Keywords: Urban rail transit; forecast maintenance; equipment fault cluster; decision tree; monitor data mining.

\section{ANALIYSIS OF MAINTENANCE}

As for various electrical and mechanical equipment serves as the crucial components of daily operation of Urban Rail Transit (URT), besides of the infrastructures like rails or vehicles. Today, in order to reasonably maintain assets and improve their utilization, URT enterprises have widely adopted the lifecycle maintenance management, which covers all stages since equipment procurement, entering the asset library, installation, status supervision, maintenance, until damage or scrapped exit. With extension of regular management, the task of monitor equipment fault and identifying potential failures are treated as the important work for daily maintenance [1].

The maintenance modes often include repair for failure, keep using time, and situation monitor, to decide which process or stage should be put in. Especially, actual situation is necessary for maintenance plan because of those devices related to service life period. Some relevant research presented this. Liu presented intelligent maintenance decision support model of equipment management based on risk, with proactive and default strategy of RCM [2]. Cheng described that the preventive maintenance is effective based on status, as it captures system situation and makes out appropriate maintenance strategy [3]. Nakagawa introduced unlimited time field into cyclical preventive maintenance, and assumed that failures obviously increase with time to discover the most optimal intervals and times of the preventive maintenance [4]. Han put forward the concept of equivalent military age, which built relations between recurrence failure rate and preventive maintenance cycle, and presented the equipment fault time is comply with Weibull distribution, also took an example to construct maintenance optimal model of finite time interval with the reliability constraints [5]. Mohammed proposed that adopting the same basic cycle repair in traditional maintenance easily leads to excess resources and inadequate maintenance repairs, so it is essential to combine plan with monitoring the failure characteristics of the device itself and the health status to make optimal decision [6]. Mao found that the rate of system failure is closely related to specific forms of failure distribution in repair intervals [7]. Thus, adjusting to appropriate time for preventive maintenance, through targeted selection of different types of equipment, the optimization results will be closer to actual requirement. Although preventive maintenance often recovers device function and reduces the failure rate, the equipment or system can't back to original status and performance. Recently, RCM is used to build a 
risk matrix and logic decision to determine risk level, failure mode and equipment essentiality through analyzing the fault rate and the severity, and recently is applied to establish the strategy of preventive maintenance based on reliability and economy $[8,9]$.

Thus, to find potential problem or risk of equipment and systems, through collecting a large amount of monitor or alarm data of failures and mining, is becoming as an essential solution for maintenance of URT, also it will be a main direction in the future.

\section{CHARACTERISTIC OF MAINTENANCE}

The maintenance of URT often points to repair all sorts of equipment and electrical mechanical system, and its operation includes inspection, routine maintenance, parts repair, special transformation etc. There are special characters in different stages.

\subsection{MULTI-MAJORITY CROSS}

The comprehensive maintenance covers mechanical and electrical equipment, like vehicles, track and various related systems. The electrical equipment is often regarded as one of the most crucial elements in URT operation, whose situation and mode can be monitored, e.g. real-time monitoring of stray power current, status supervision of automation system, and bearing temperature detection of vehicle running gear. The collection also includes various type of off-line mode and the handheld equipment of condition monitoring like detection tool of vehicle wheel wear. The results of online and offline monitoring will be pooled into a unified monitoring system for maintenance.

General maintenance of vehicles involves several processes, including the train inspection, month repair, scheduled repair, and plant maintenance. Corresponding to the initial failure, occasional failure, and broken down stage, the repair cycle is matched on certain parameters of state, walk miles and age of the vehicle. Currently, the maintenance is mainly for plan repair, and state repair acts as supplementation [10], which consists of systems, subsystems, devices, components, parts, spare parts. Therefore, the mechanical and electrical system and equipment in situation of multi-major cross are focus.

\subsection{LIMITATION OF PLANNED REPAIR}

Operators usually use system to carry out planned repair task, which can be divided into two types, regular preventive repair and corrective maintenance. The former aims to detect the part mistakes and make the equipment back to initial state, whereas the purpose of the latter is to bring equipment to the state before failure by repairing broken down devices. Moreover, simple maintenance system is just front-end applications, which not relate to asset maintenance records of Enterprise Asset Management (EAM) System and without monitor data mining analysis, so it is unable to support prevention and intelligent predictive maintenance.

\subsection{CLOSELY CORRELATION OF MONITOR AND MAINTENANCE}

The maintenance plan needs data source from supervision systems, including equipment state, fault data, repair data, technical documents and standardized failure records. Those of faulty data describe the features, such as mode, location, reason, happened time etc. The repair data supplies begin and end time of fault, and effect like safety, environment, production, and cost. Then the statistics indexes like Mean Time between Failure (MTBF), Mean Time to Repair (MTTR), Potential Failure-failure interval (P-F), will be calculated. Thus, the establishment and evaluation of maintenance should be coordinated with supervision systems.

\subsection{PERTINENCE OF COMPREHENSIVE MAINTENANCE}

Nowadays, the equipment can be monitored by various supervision systems, such as Power Supervision Control And Data Acquisition System (PSCADA), Building Automation System (BAS), Fire Alarm System (FAS), Automatic Train Supervision (ATS), Passenger Information System (PIS), Automatic Fare Collection System (AFC), Platform Screen Door system (PSD), etc. [11], in respective major concerns. So that we take all of them as the goal objects to adopt preventive maintenance is impossible and unreasonable. At first, the strategy is to choose crucial equipment that may lead to damage or risk for normal operation of URT as the focus in order to put into range of related maintenance management.

\section{DECISION MAKING SUPPORT FOR INTELLIGENT MAINTENANCE}

To build strategic maintenance, at first analysis of data source and operation process are necessary. Aiming to find the intensive objects that causing damage and high frequency repair, the results from data mining can help to discover the common rules for system failure [12, 13]. We presented an approach of preventive and predictive maintenance to solve bottleneck problems and obtain essentially 
improvement, called intelligent decision for maintenance.

\subsection{MAINTENANCE PROCESSING}

The maintenance operation can be generalized as follows:

(1) Tracking and recording: e.g. equipment procurement, receiving, distribution, installation, testing, decommissioning, and change.

(2) Fault records: key note includes question code, fault code, reason code, execution code, and device use record.

(3) Repair management: program, organization, value, quality, and outsourcing.

Besides basic maintenance work, there are three sub-process: equipment resume update from EAM, equipment status collection from supervision system, data mining of preventive maintenance associated with high frequency fault identification.

The interfaces of monitor system related to maintenance management include:

(1) Interface of EAM

EAM contains all asset information, such as device history, state record, inventory and procurement transaction, employee, certification, calendar etc. To achieve integrated management history of device, standard device ID for retrieval and data source for crucial equipment of repair are needed.

(2) Interface of supervision systems

It is used to collect equipment status and fault information, in order to analyze a large scale history alarm, which will output programs for finding potential inefficient equipment and calculate equipment failure rates.

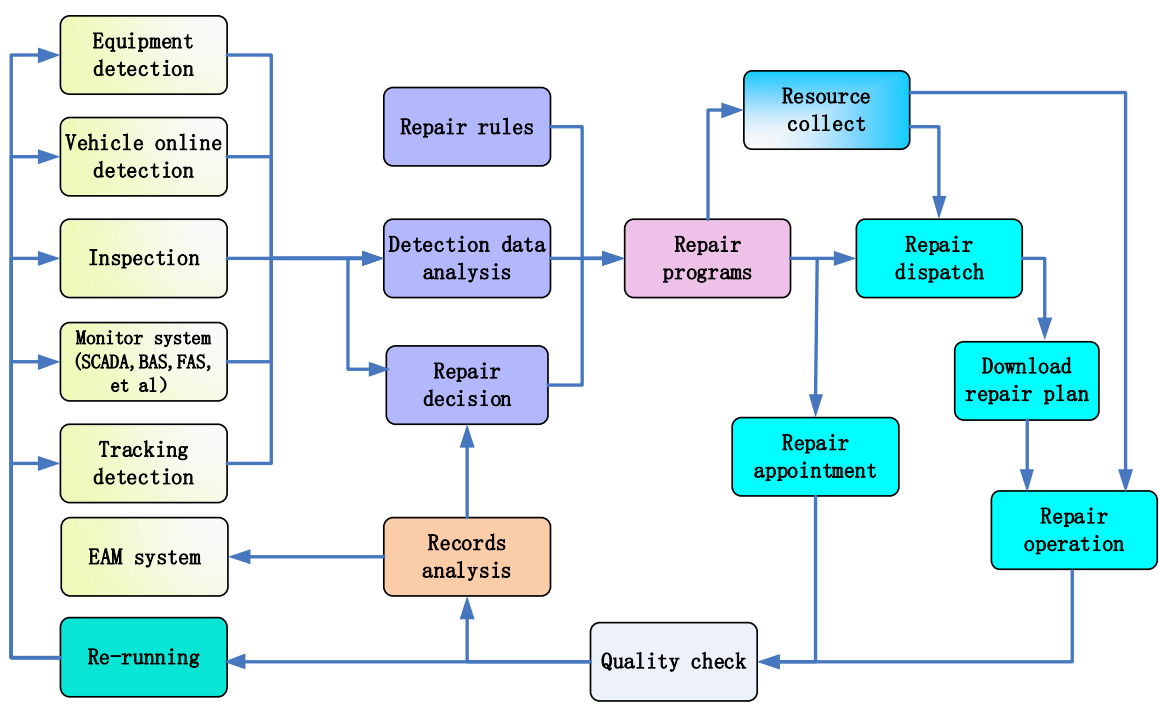

Fig. 1 - Relation of repair and monitor systems

(3) Interface of data mining with high rate fault

To make an effective plan, the device attribute records and alarm data were employed to find equipment fault type with higher rate and related subsystems. Predisposing data was the first step to build the cluster model for selected objects, and then we identified the rules and made reasonable evaluation.

\subsection{COMBINATION OF PREVENTIVE MAINTENANCE AND INTELLIGENT DECISION}

The planned maintenance is often used after fault appeared, whereas the preventive maintenance serves as the main way. We divided the preventive maintenance into two types based on time or on situation. The first type required repair cycle was relatively fixed, and another type set repair strategy of check, monitor and emergency. The measure is used to improve the utilization ratio and reliability of equipment $[14,15]$.

(1) Preventive repair: It aims to find the most suitable maintenance cycle, through overhaul cycle of data mining and adjustment, in order to avoid excessive repair resulted in waste of cost.

(2) Predictive repair: It performs inspections of key equipment, priority processing, and mean value analysis, through dynamic and static examination methods to evaluate equipment reliability and quality of maintenance.

(3) Faulty repair: It establishes the fault system that records the properties and details of failure to improve fast repair ability and further provide some advice for technological transformation.

Thus, we defined different stage of intelligent maintenance as three dimensions: process, content, and value. Besides, from the view of factors like cost 
and value, the following qualifications of equipment selection was presented for forecast and preventive repair.

a. The running state has greater impact on trains or operating cost.

b. The devices have relatively more monitoring or state detection ways available for analysis.

c. The equipment has historical data to support dynamically manage the maintenance cycle.

\section{PRE-SELECTED MAINTENANCE MODEL}

How to find the key equipment that influences operation based on data mining for hundreds of equipment? That is a problem of decision making for preventive maintenance that we confronted at first.

\subsection{HIGH FREQUENCY REPAIR CLUSTERING}

In order to achieve preventive repair target objects from disorderly and numerous alarm records, the common characteristics should be identified and form a cluster. We chose attribute variables and fault variables of equipment, applying K-means Clustering theory [16-18], we built faulty clustering model based on different classification trend and statistical purposes. Also, after finishing data preprocessing by adopting SPSS tools, the clustering was attained according to the features of fault.

At first, define a fault class collection $C=\{P, B, F, \mathrm{~A}, S, C, D\}$ according to different monitor systems, $P$ means PSCADA, $B$ means BAS, $F$ means FAS, $A$ means ATS, $S$ means PIS, $C$ means AFC, $D$ means PSD. Take fault equipment as the object of each data set, including attributed sub-system, the single device, parts and components. We put collection of BAS system as an example to describe the process of clustering. Assign object in $B$ into $k$ clusters $\left\{\beta_{1}, \cdots, \beta_{k}\right\}$, meet $1 \leq k, j \leq k, \mathrm{~s} \beta_{i} \subset B$, and $\beta_{i} \leq \beta_{j}=\Phi$. The object function set high similarity within clusters and low similarity between clusters, to obtain the same type of fault cluster in the most relevant and biggest difference with other objects. Use the centroid to represent the cluster $\beta_{i}$ and define mean value of objects in cluster. Define $\operatorname{dist}(x, y)$ is the distance between $x$ and $y$, which represents the device $x \in \beta_{i}$ different from the delegate $b_{i}$, and quality of cluster $\beta_{i}$ is measured by variation of inter clusters, expressed as

$$
E=\sum_{i=1}^{k} \sum_{x \in b_{i}} \operatorname{dist}\left(x, b_{i}\right)^{2}
$$

The algorithm is given as follows:

Step 1: Filter the fault data and remove irrelevant records on the basis of the business and related equipment properties. Then regard them as the independent cases and build fault collection $B()$ with the fault similarity.

Step 2: Random pick out $k$ objects from $B()$, every object is calculated original mean value and as the center of cluster, and the distance $\operatorname{dist}\left(x, b_{i}\right)$ from the center of others is calculated to decide how to allocate to the most relevant fault class of similar clusters.

Step 3: Use the K-means iterative algorithm, take the improvement of cluster variation as the goal.

Step 4: For each cluster, assign previous iteration object on the cluster to compute new mean value, and pick updated mean as the new center of the cluster to reallocate all objects.

Step 5: Execute the process until the distribution turn to stable.

The principle of calculation:

(1) In order to avoid this method to converge global optimization, frequency sorting was employed based on statistics of fault in a certain period of time. And 5\% of fault classes was picked as the initial clusters' center in turn, repeatedly running the algorithm.

(2) Calculate the complexity $O(n k t)$, the main fault types were identified to assess the rate. Take $k$ and total number $n$ of objects, meet $k<<n$, and iteration times $t$, meet $t<<n$, to ensure the validity of calculation results.

Comparing the fault frequency of various special equipment, we chose the equipment whose failure rate reached a certain percent ( $80 \%$ in this project), listed in high-frequency repair classes, as the key objects of repair for forecast, after running after a certain period.

\subsection{PRE-SELECTED CLASS OF REPAIR BASED ON DECISION TREE}

Based on clustering process, we found those equipment with higher fault rate and treated as objected repair types to pay attention. But the goal is not limited to this, according to these preselection classes; it is easy to identify the relationship between the device and the subsystem. And further we deduced the similar types of failure may cause linkage failure, and made out the early warning of equipment and subsystems. According to this reverse derivation of the rules, it is appropriate to use the decision tree to sum up its rules to identify the potential needs of the maintenance of equipment.

Thus, the decision tree induction strategy was adapted to help us derive rules and characteristics of 
key repair objects to identify pre-selected class, which expanded range of potential repair points [1921]. The method of anti-direction decision tree induction means given a tuple $x$ with unknown label class, whose attribute values are tested in decision tree. Following a path from the root to the leaf node, it is stored in class prediction of the tuple, then the classification rules are transformed from decision tree. Because of the extracted rules with disjunctive relation, it is mutually exclusive or exhaustive. Corresponding to any two rules may not be produced by the same tuple trigger, any combination of attribute values have a rule, so that the disorder of rules recognition is secured. The advantage of this method is to construct the classifier without require domain knowledge or parameter set, also not depend on the correlation among devices in the highfrequency repair clustering. Moreover, it suits detective knowledge discover, and can be used as a supplement for cluster analysis. The deduction algorithm of the decision tree rule is described as follows:

(1) Data pre-processing. The attribute selection: set a data partition $D$ for a training set of labeled class tuple, assuming that its attributes have $m$ different values, and define $m$ class $C_{i}(i=1, \cdots, m)$. Set $C_{i, D}$ as a collect of tuple $C_{i}$ in $D,|D|$ and $\left|C_{i, D}\right|$ are the number of tuple $D$ and $C_{i}, D$ respectively.

(2) Create node $N$. According to the clustering algorithm, the fault class cluster $\beta()$ can be gotten. Set $C_{0}=\beta$, means a fault feature class assigned to class $C_{i}$.

(3) Assume the tuples in $D$ are all in class $C$, $N$ serves as leaf node, marked as $C$. If the attribute table is empty, $N$ is also treated as a leaf node, marked in the majority class $D$.

(4) Let $D_{j}$ as a collect of tuples to meet output $j$ in $D$. If $D_{j}=\Phi$, then add a leaf to node $N$ and marked in the majority class $D$, else add a node returning from $C_{i}$ to $N$, and iterate to traverse the entire training set.

The crucial step lies in rules deduction in this algorithm. Create a rule for each path from the root to leaf nodes, with splitting criterion along each path to form its antecedent, and the leaf nodes storing class prediction will form the rules consequents. The equipment of mainly fault class and its property division, preliminary determined by clustering algorithm, provide rules as constraint of rule antecedent for their production.

Moreover, to ensure the effectiveness of the primary results, we used the method of testing the model of decision tree to calculate indexes for performance evaluation, including the accuracy rate, hit rate, and recall rate. The last two rates were calculated by coincidence matrix node of the crossing table, according to the estimation value and actual value, through comparing the chosen period of failure data with continuous period of real faults.

\subsection{EVALUATION MODEL AND INSPECTION}

In order to improve the reliability of pre-class, the result was conversed and further verified. The discrete variables of sign was transmitted to continuous variables according to given rules, also, they were used to established prediction model, using Neural Network method to test results.

Define discrete variable of high frequency fault class $V_{\text {repair }}()$, and convert pre-selected class of preventive repair to continuous variable $V_{\text {repair_score }}()$.

Using the Neural Network model, we obtained a preselected probability score for decision tree model, then the score results were calculated for each class to judge whether as a repair class. Judging rules are designed as follows:

If $N-V_{i} \geq 0.5$, then estimation fault value is 1 , master degree is $2 \times\left(N-V_{i}\right)-0.5$.

If $N-V_{i}<0.5$, then estimation fault value is 0 , master degree is $2 \times\left(N-V_{i}\right)+0.5$.

Comparing the scoring results with anti-direction class by the decision tree based on clustering analysis, then the part of overlap was for output.

\section{IMPLEMENTATION}

On the basis of repair operation of URT, the Maintenance Management System (MMS) was developed with the functions including asset management, maintenance management, equipment management, repair supervision, etc. It is essential that define a standard process of maintenance to realize decision management of preventive repair.

Take a metro rail line that has operated for some period as an example, collecting monitor data of equipment fault for 6 months. First of all, we identified the type of fault by clustering, then used model of high frequency repair pre-selected class to create aim class, so the crucial objects for preventive maintenance were achieved. The data disposing process is expressed in following picture.

The data collect was chosen to build fault repair clustering model, including:

(1) Total of clusters with similar fault cycle of equipment.

(2) Total of clusters that happen to continuous multiple faults after first repair.

(3) Total of clusters that can run for some periods after repair. 


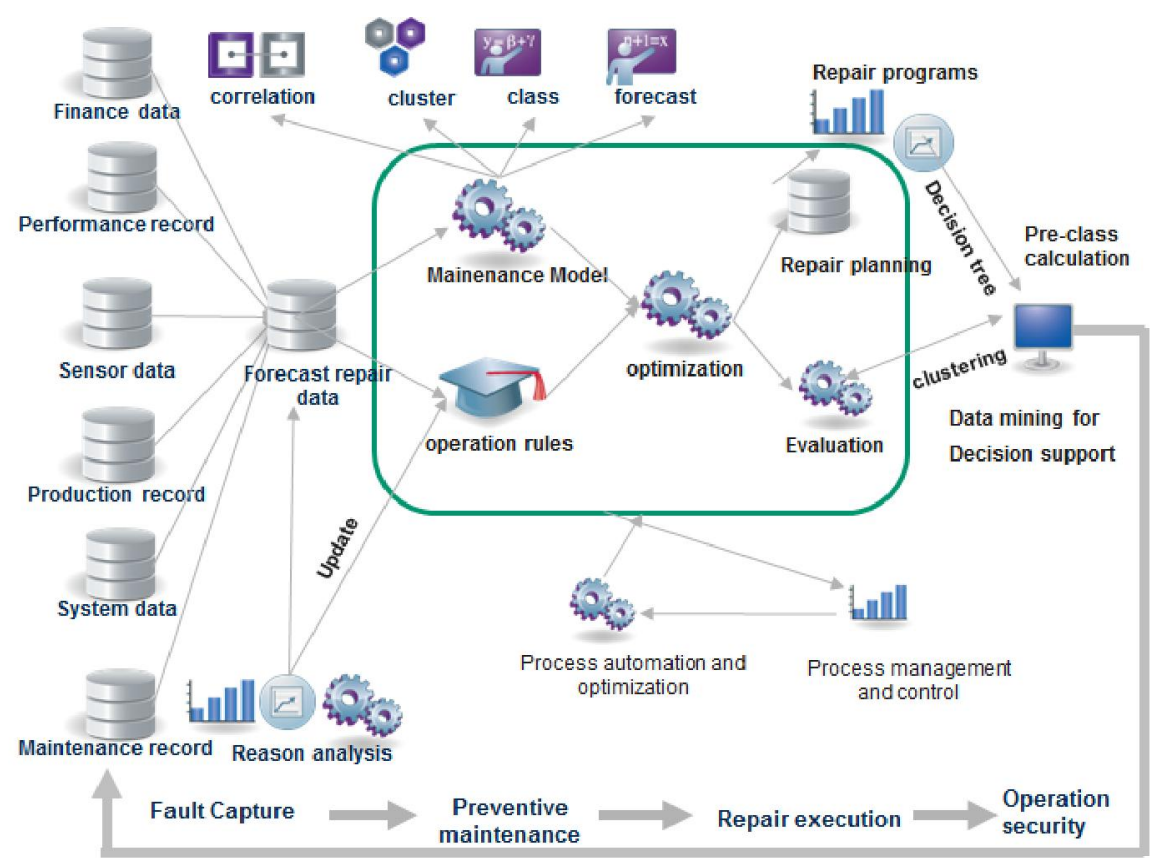

Fig. 2 - Flow chart of data preparation

The distribution and comparison of each group characteristics has achieved from clustering model.

The details are showed as following table.

Table 1. Distribution of fault cluster

\begin{tabular}{|c|c|c|}
\hline cluster & Percent(\%) & value \\
\hline 1 & 0.2634 & 926 \\
\hline 2 & 18.4231 & 64774 \\
\hline 3 & 0.0023 & 8 \\
\hline 4 & 11.8405 & 41630 \\
\hline 5 & 69.4708 & 244253 \\
\hline
\end{tabular}

Owing to the data flow and the rule set, we derived high-frequency repair rules and pre-selected class. The experiments covered 3 million records of fault of 5 monitor systems with 30 stations, all of which were saved in MMS database and the results can be seen in Fig. 3.

From the result of evaluation, the overall accuracy rate of model reached $96.74 \%$ of test data, the result met the requirements. Besides, the class variables of repair device were identified to correlate with high frequency fault by way of discrete variable, in order to find main related factors of preselected class, through introducing Neural Network of data mining method, and further to the propensity score of high frequency failure was assessed.

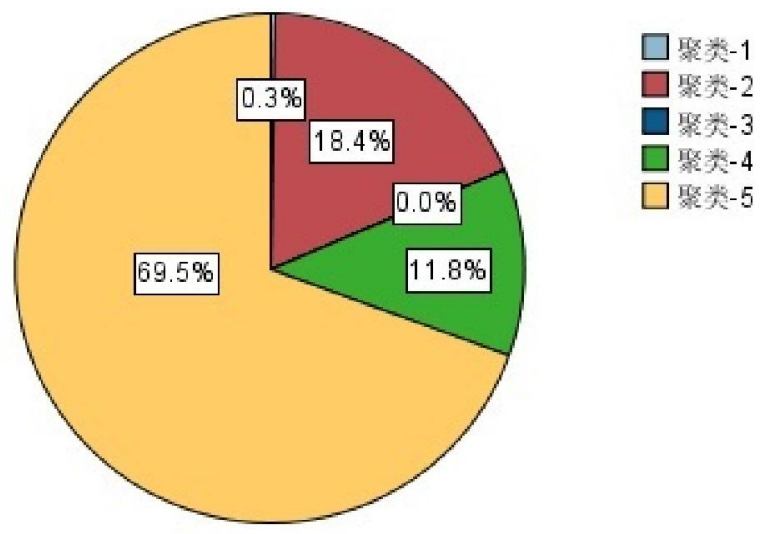

Fig. 3 - Percent of fault clusters

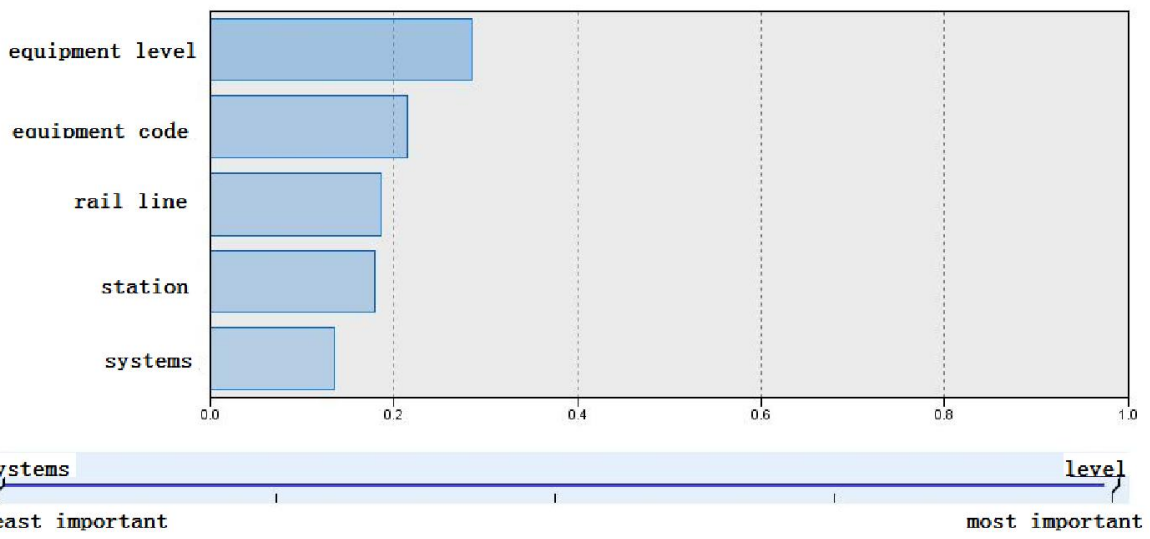

Fig. 4 - Assessed score from Neural Network method 


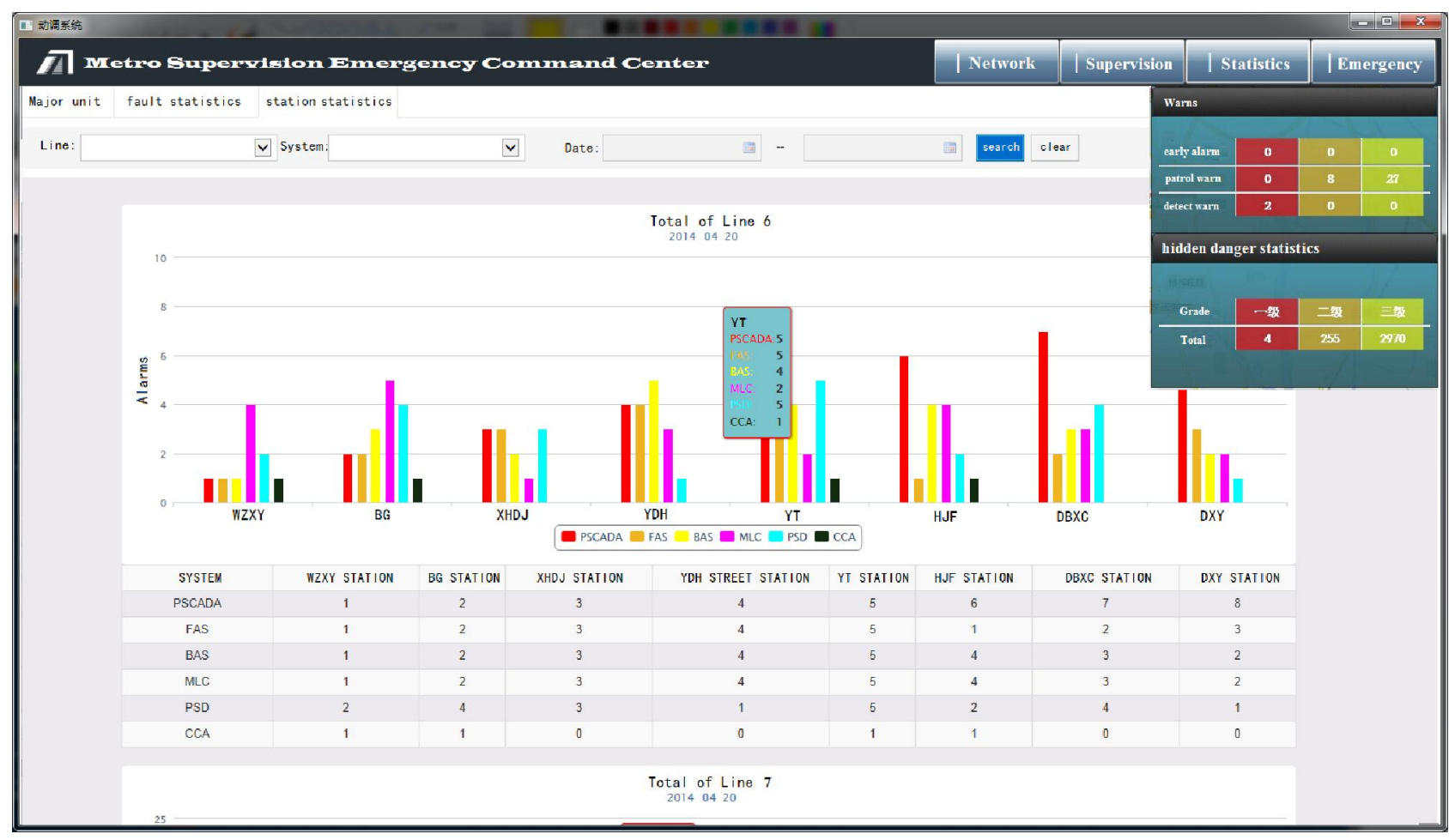

Fig. 5 Pre-selected class factors algorithm and fault statistics

Choose the clustering data set as the data source, the neural network model was introduced, and the device fault class preselected variable was transformed into a continuous variable based on the alarm time, using the IBM SPSS tool software. Define the transformation rule of the symbolic discrete variable $V_{\text {repair }}()$ of fault repair into a continuous variable $V_{\text {repairvalue }}()$ of alarm evaluation, Then the equipment is fault repair a converted to the alarm evaluation value $b$ expressed as

if $V_{\text {repair }}()=1$, then $V_{\text {repairvalue }}()=100.0$; if $V_{\text {repair }}()=0$, then $V_{\text {repairvalue }}()=0.0$

When taking goal variable as continuous variable for fault forecast model with Neural Network method, the calculation result was transformed, and we set forecasting certainty extent by following way:

If $V(i)-V_{\text {repairvalue }} \geq 0.5$, then forecast value $f=1$, and the extent is: $2 \times\left(V(i)-V_{\text {repairvalue }}\right)-0.5$.

If $V(i)-V_{\text {repairvalue }}<0.5$, then forecast value $f=0$, and the extent is: $2 \times\left(V(i)-V_{\text {repairvalue }}\right)+0.5$.

Comparing the decision tree by using discrete variables with neural network with regarding object variable as continuous variable, result of high frequency pre-selected class has appeared consistent performance.

According to calculation before, we obtained the results from these example data, which would be the crucial equipment and subsystems used to maintain and repair in advance, shown as in Table 2.
Table 2. Results of chosen class for repair

\begin{tabular}{|c|c|c|c|}
\hline system & cluster & equipment & $\begin{array}{l}\text { Link } \\
\text { index }\end{array}$ \\
\hline \multirow{9}{*}{ PSCADA } & \multirow{3}{*}{ breaker } & 110KV switch & 0.060 \\
\hline & & 1500KVswitch & 0.093 \\
\hline & & 35KV switch & 0.023 \\
\hline & \multirow[b]{2}{*}{$\begin{array}{l}1500 \mathrm{~V} \text { DC into } \\
\text { the cabinet }\end{array}$} & Negative abinet & 0.007 \\
\hline & & $\begin{array}{l}201,202,211,21 \\
2,213,214 \text { switch }\end{array}$ & 0.122 \\
\hline & $\begin{array}{l}\text { protects the total } \\
\text { failure report }\end{array}$ & $\begin{array}{c}1500 \mathrm{~V} \\
1 \# \text { Route201 }\end{array}$ & 0.023 \\
\hline & $\begin{array}{l}\text { protects the total } \\
\text { failure report }\end{array}$ & $\begin{array}{c}1500 \mathrm{~V} \\
1 \# \text { Feeder211 }\end{array}$ & 0.069 \\
\hline & $\begin{array}{c}\text { Straight circuit } \\
\text { breaker trip signal }\end{array}$ & $\begin{array}{c}1500 \mathrm{~V} \\
\text { 1\#Route201 }\end{array}$ & 0.210 \\
\hline & $\begin{array}{c}\text { Feeder circuit } \\
\text { breaker trip signal }\end{array}$ & $\begin{array}{c}1500 \mathrm{~V} \\
1 \# \text { Feeder213 }\end{array}$ & 0.392 \\
\hline \multirow{8}{*}{ BAS } & FDS & sewage pump & 0.001 \\
\hline & CWS & Chillers & 0.001 \\
\hline & BAS & $\begin{array}{c}\text { Multi-air } \\
\text { conditioning }\end{array}$ & 0.032 \\
\hline & FDS & $\begin{array}{l}\text { Water supply } \\
\text { and drainage }\end{array}$ & 0.006 \\
\hline & TVS & Track fan & 0.000 \\
\hline & PVS & ventilator & 0.262 \\
\hline & BAS & $\begin{array}{c}\text { Water collection } \\
\text { wells }\end{array}$ & 0.002 \\
\hline & PVS & Air condition & 0.695 \\
\hline \multirow{4}{*}{ FAS } & alarm & Fire alarm & 0.678 \\
\hline & \multirow[b]{3}{*}{ Equipment failure } & detectors & 0.188 \\
\hline & & Fire Pump & 0.115 \\
\hline & & $\begin{array}{l}\text { Temperature } \\
\text { sensing cable, } \\
\text { sensitive fiber }\end{array}$ & 0.020 \\
\hline
\end{tabular}


From the results, the correlation of the failure is weak in PSCADA system and its subsystem, whereas a class of coordination subsystems in BAS and FAS system exhibitted a high proportion index of device failure

In summary, more equipment failure in the subsystem environment is still showing a higher risk of whole system, the distribution showed the compliance with the nature and the degree of the alarm system, which indicated that they are little affected by other equipment with the impact of interference. So the maintenance personnel focus on that higher failure rate of monomer equipment or subsystems.in the investigation of hidden dangers. In contrast, the performance of the integrated alarm value of the associated subsystem is the opposite. When the high-frequency faulty devices with different monitoring types worked together, if the correlation degree was not strong or did not interfere with the associated system, the warn degree is low signal; but if the impact of the early warning signal performance is stronger, the more dangerous appeared, even led to damage of whole system. Therefore, through the complex alarm rules, can find a strong degree of relevance of the object, as a major hazard monitoring list to support preventive and predictive maintenance reasonably.

\section{CONCLUSION}

The data mining and analysis of supervision systems showed effective solution for maintenance decision. Concerning characteristics of mechanic equipment fault of URT, the approach of this paper presented identified main equipment type for preventive maintenance, through adopting clustering and decision tree rules. From the view of discrete alarm data and continuous variables to evaluate the pre-selected class, the effectiveness of results were proved. Furthermore, on the condition of dynamical adjust of repair cycle, it self-suitably converted repair these class and applied record to update plan of the preventive maintenance. The method has been put into implementation of the maintenance management system, serving as a convenient and visional decision tool for URT operators.

\section{REFERENCES}

[1] M. Zhang, "Decision approach of maintenance for urban rail transit based on equipment supervision data mining," in Proceedings of the International Conference on Intelligent Data Acquisition and Advanced Computing Systems: Technology and Applications(IDAACS), Warsaw, Poland, September, 24-26, 2015, pp. 376-380.
[2] W. Liu, Q. Wang, J.Gao et al., "Reliabilitycentered intelligent maintenance decisionmaking model," Journal of Beijing University of Technology, vol. 38, issue 5, pp. 672-677, 2012.

[3] T. Nakagawa, "Sequential imperfect preventive maintenance policies," IEEE Transactions on Reliability, vol. 37, issue 3, pp. 295-298, 1988.

[4] B. Han, X. Fan, D. Ma, "Research on the optimal preventive maintenance policy based on reliability constraints," Chinese Journal of Mechanical Engineering, vol. 39, issue 6, pp. 102-105, 2003.

[5] M. Sbihi, C. Varnier. "Single-machine scheduling with periodic and flexible periodic maintenance to minimize maximum tardiness," Computers \& Industrial Engineering, vol. 55, issue 4, pp. 830-840, 2008.

[6] Z. Mao, B. Song, G. Pan, "Optimal system preventive maintenance model of different preventive maintenance period," Fire Control \& Command Control, vol. 35, issue 3, pp. 5860, 2010.

[7] R P. Thomas, Total Productive Maintenance (TPM) Concepts and Literature Review [EB/OL], 2004, [Online]. Available: http://www.brooks.com/tmp/2110.pdf.

[8] D. Ting, Computer Maintenance Management System, ITIS Mechanical Industry Research Laboratories, October 2001.

[9] Q. Wen. "On integrated maintenance system of shanghai URT," Urban Mass Tranist, issue 4, pp. 2-11, 2008.

[10] M. Zhang, F. Wang, P. Li, "The platform of network operation decision making and emergency for urban rail transit," China Academy of Railway Sciences, vol. 33, issue 1, pp. 113-120, 2012.

[11] S.-F. Fang, W. Lu, "Research on a maintenance intelligent decision support system," Journal of Systems Science and Information, vol. 12, pp. 53-59, 2001.

[12] S. Madan, W. K. Son, K. E. Bollinger, "Applications of data mining for power systems," in Proceedings of the IEEE Canadian Conference on Electrical and Computer Engineering, St. John's, Canada, 1997.

[13] A. Famili, S. Létourneau, "Monitoring of aircraft operation using statistics and machine learning," in Proceedings of the IEEE International Conference on Tools with AI'99. Chicago, USA, November 9-11, 1999, pp. 279286.

[14] M. Fan, X. Meng, Data Mining Concepts and Techniques, 3rd edition, China Machine Press, Beijing, 2013, pp. 298-303. 
[15] M. Traore, A. Chammas, E. Duviella, "Supervision and prognosis architecture based on dynamical classification method for the predictive maintenance of dynamical evolving systems," Reliability Engineering \& System Safety, vol. 136, pp. 120-131, 2015.

[16] G. Cardoso, J. G. Rolim, H. H. Zurn, "Identifying the primary fault section after contingencies in bulk power systems," IEEE Trans. on Power Delivery, vol. 23, issue 3, pp. 1335-1342, 2008.

[17] L. P. Khoo, L. Y. Zhai, "A prototype genetic algorithm-enhanced rough set-based rule induction system," Computers in Industry, vol. 46, pp. 95-106, 2001.

[18] J. H. Friedman, J. J. Meulman, "Clustering objects on subsets of attributes," Journal Royal Statistical Society Series B Statistical Methodology, vol. 66, issue 4, pp. 815-849, 2004.

[19] A. Bobbin, I. Portinale, M. Minichino, et al., "Improving the analysis of dependable systems by mapping fault trees into Bayesian networks," Reliability Engineering and System Safety, vol. 71, pp. 249-260, 2006.
[20] J. Cheng, D. Bell, W. R. Liu, "Learning Bayesian networks from data: An efficient approach based on information theory," Artificial Intelligence, vol. 137, issue 2, pp. 4390, 2002.

[21] S. Kim, M. G. Tadesse, M. Vannucci, "Variable selection in clustering via Dirichlet process mixture models," Biometrika, vol. 93, issue 4, pp. 877-893, 2006.

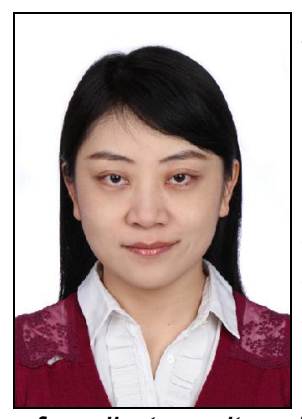

Zhang Ming, Ph.D, Research associate, China Computer Society (CCF) member. Graduated from Tongji University, doctorate in transportation planning and management. The main research directions include the intelligent system technology of Urban rail transit, safety and emergency planning. In the field of rail transit, charge and participate in many research projects like National Natural Science Fund, National Science and Technology Support program, also joined the engineering implementation. 